\title{
Planar Superconductor-Normal-Superconductor Josephson Junctions in $\mathrm{MgB}_{2}$
}

\author{
G. Burnell*, D.-J. Kang*, H.N. Lee $\dagger$, S.H. Moon $\dagger$, B. Oh†, and M.G. Blamire* \\ *Dept. Materials Science and IRC in Superconductivity, University of Cambridge \\ Pembroke Street, Cambridge CB2 3QZ, UK \\ $\dagger$ LG Electronics Institute of Technology, Seoul 137-724, Korea
}

Since the discovery of superconductivity in $\mathrm{MgB}_{2}{ }^{1}$ considerable progress has been made in determining the physical properties of the material, which are promising for bulk conductors ${ }^{2-5}$. Tunneling studies ${ }^{6-9}$ show that the material is reasonably isotropic and has a well-developed s-wave energy gap $(\Delta)$, implying that electronic devices based on $\mathrm{MgB}_{2}$ could operate close to $30 \mathrm{~K}$. Although a number of groups have reported the formation of thin films by post-reaction of precursors $^{10-14}$, heterostructure growth is likely to require considerable technological development, making single-layer device structures of most immediate interest. $\mathrm{MgB}_{2}$ is unlike the cuprate superconductors in that grain boundaries do not form good Josephson junctions, and although a SQUID based on $\mathrm{MgB}_{2}$ nanobridges has been fabricated ${ }^{15}$, the nanobridges themselves do not show junction-like properties. Here we report the successful creation of planar $\mathrm{MgB}_{2}$ junctions by localised ion damage in thin films. The critical current $\left(I_{C}\right)$ of these devices is strongly modulated by applied microwave radiation and magnetic field. The product of the critical current and normal state resistance $\left(I_{C} R_{N}\right)$ is remarkably high, implying a potential for very high frequency applications.

Our film deposition technique has been described elsewhere. ${ }^{12}$ Briefly, B films were deposited at room temperature on to (0001) sapphire substrates by electron beam evaporation and then ex-situ annealed in a $\mathrm{Mg}$ vapour at $850^{\circ} \mathrm{C}$ for 30 minutes to produce $\mathrm{MgB}_{2}$ films with a final thickness of $100 \mathrm{~nm}$ and a critical temperature $\left(T_{C}\right)$ of $36 \mathrm{~K}$. We deposited a $20 \mathrm{~nm}$ Au film onto the films by dc magnetron sputtering in order to protect the $\mathrm{MgB}_{2}$ from contact with water during subsequent processing. Tracks and contact pads were patterned in the bilayer film using standard photolithography and broad beam Ar-ion milling. The $T_{C}$ of the patterned tracks was approximately $35 \mathrm{~K}$. In order to fabricate the junctions, the film was then transferred to a focused ion beam system (FIB) (Philips-FEI Inc. FIB 200). Chips were wirebonded to enable the resistance of the tracks to be monitored during the FIB milling process. ${ }^{16}$ The barrier was defined by writing $50 \mathrm{~nm}$ wide cuts across the width of tracks using a $4 \mathrm{pA} 30 \mathrm{kV}$ Ga ion beam. The depth of the cut was calibrated by comparing the cut time to that required to completely sever a track; since the Au mills more than order of magnitude faster than the $\mathrm{MgB}_{2}$ it could be ignored in calibrating the cut depth. Compared to other materials used to fabricate SNS junctions using this technique, ${ }^{17} \mathrm{MgB}_{2}$ offers the advantage of a relatively low milling rate and hence excellent depth control due to the low atomic mass of its constituents and high melting point.

The devices were measured in dip probes placed in ${ }^{4} \mathrm{He}$ storage dewars. The probes are equipped with coils to provide a magnetic field perpendicular to the substrate plane, and with a microwave antenna. The inset to Fig. 1 shows the resistance against temperature for two tracks on the same substrate, one in which no junction was made (a) before placing in the FIB and (b) after the FIB stage, and 
another track (c) in which a junction had been fabricated. The FIB process clearly has no significant effect on unpatterned films. In case (c) the track with a junction shows an increase in resistance below $125 \mathrm{~K}$ and a pronounced 'foot' below the main film $T_{C}$ before becoming completely superconducting at $25 \mathrm{~K}$ (the absolute resistance is lower than $(\mathrm{a} / \mathrm{b})$ due to different track geometries).

Below the $T_{C}$ of the devices, non-hysteretic, resistively shunted junction (RSJ)-like $I$ - $V$ characteristics were obtained. In Fig. 1 we show the current vs voltage characteristics at several temperatures for a device with a nominal $75 \%$ cut in a $2.5 \mu \mathrm{m}$ wide track. Figure 2 shows the temperature dependence of $I_{C} R_{N}$ for the same device as Fig. 1. As shown in the inset, $R_{N}$ (measured from a linear fit to the $20 \%$ highest bias in the $I-V$ ) is approximately constant at $3.5 \pm 0.5 \Omega$ over the entire temperature range; this is consistent with the additional resistance upturn in the normal resistance-temperature data extrapolated to $0 \mathrm{~K}$ and implies a relatively high barrier resistivity of order of $200 \mu \Omega \mathrm{cm}$.

Application of an out of plane magnetic field $(B)$ to the junctions resulted in a substantial modulation of the critical current. Figure 3 shows $I_{C}(B)$ at $18 \mathrm{~K}$ for the same device as in Fig. 2. The apparent incomplete suppression is partly due to the fixed voltage criterion used to determine $I_{C}$, however there is genuinely a remnant critical current for all applied fields. At lower temperatures the minimum $I_{C}$ is larger relative to the maximum value which suggests that the barrier is to some degree nonuniform. In a substantially wider junction we observed a rapid SQUID-like $I_{C}(B)$ modulation attributed to a strongly non-uniform barrier; this is probably a consequence of the surface roughness of the films used.

The $I_{C}(B)$ modulation clearly differs substantially from the ideal Fraunhofer pattern and is very strongly hysteretic. However, the form is identical to that observed in planar high $T_{C}$ junctions in which the hysteresis has been successfully modelled on the basis of Abrikosov vortex penetration into the electrodes ${ }^{18}$. Magneto-optic imaging of thin films has shown that polycrystalline $\mathrm{MgB}_{2}$ films such as ours trap flux at very low fields ${ }^{19}$. Mitchell et al. ${ }^{18}$ show that in such circumstances the position of the first minimum provides the most reliable estimate of the magnetic penetration depth $(\lambda)$. Ignoring flux focusing effects, which are likely be small for a junction of this size, we obtain a value for $2 \lambda+d$ of $331 \mathrm{~nm}$. Assuming that the electrical length of the barrier is the same as the defined cut width gives a value of $\lambda$ of just over $140 \mathrm{~nm}$. This is very similar to estimates obtained elsewhere by different techniques. ${ }^{20-22}$

Complete suppression of the $I_{C}$ and the formation of Shapiro steps were observed when the device was irradiated with microwaves. We observed the clearest Shapiro steps at a frequency $(f)$ of $14.96 \mathrm{GHz}$ over a temperature range of $7 \mathrm{~K}$ to $17 \mathrm{~K}$. Above this temperature range the height of the Shapiro steps was too small to observe clearly, whilst below the large $I_{C} R_{N}$ product prevented effective coupling of the incident microwave radiation to obtain a complete suppression of the $I_{C}$. In Fig. 4 we show the amplitude of the $I_{C}$ and first two Shapiro steps as a function of the incident microwave power for a temperature of $11 \mathrm{~K}$. We observed a linear suppression of the $I_{C}$, as expected for a low reduced frequency $\left(f / I_{c} R_{n}\right)$. At temperatures in the middle of the range we obtained up to four Shapiro steps on the positive and negative branches of the $I-V$ (Fig. 4 inset).

The high value of $I_{C} R_{N}(1 \mathrm{mV}$ at $4.2 \mathrm{~K})$ is consistent with that reported for break junctions in bulk $\mathrm{MgB}_{2}{ }^{23}$. The temperature dependence of $I_{C} R_{N}$ is broadly can be fitted by a solution of the Usadel equations for a structure with a one dimensional non-superconducting weak link in equilibrium with superconducting electrodes for a barrier length $(d)$ of the region of 8 times the normal state coherence length $\left(\xi_{N D}\right)$ 
implying a value for $\xi_{N D}$ of order of $6 \mathrm{~nm}^{24}$. For a comparison we also show in Fig. 2 data from a $\mathrm{YBa}_{2} \mathrm{Cu}_{3} \mathrm{O}_{7-\mathrm{d}}$ planar SNS junction with the same value of $d / \xi_{N D}$ plotted against reduced temperature ${ }^{25}$ : there is excellent agreement between the data consistent with ideal SNS behaviour, but the very different values of $I_{C} R_{N} / \Delta$ provides evidence that the two systems are rather different electronically. If we take the modal value of $\Delta$ reported in the literature $(5 \mathrm{meV})^{6-9}, I_{C} R_{N}$ for the $\mathrm{MgB}_{2}$ junction is close to the direct prediction of de Gennes, ${ }^{24}$ whereas for the $\mathrm{YBa}_{2} \mathrm{Cu}_{3} \mathrm{O}_{7-\mathrm{d}}$ junctions it is much lower, implying strong gap suppression in the electrodes. ${ }^{25}$ Direct calculation of $\xi_{N D}$ $\left(\left(\hbar v_{f} l /\left(4 \pi k T_{c}\right)\right)^{0.5}\right)$ is difficult given the uncertainty of the mean-free path $(l)$, but using a value of $4.7 \times 10^{5}$ for the Fermi velocity $\left(v_{f}\right)^{2}$, our value for $\xi_{N D}$ implies that $l$ is of the order of $5 \mathrm{~nm}$. A value of $60 \mathrm{~nm}$ for polycrystalline superconducting $\mathrm{MgB}_{2}$ has been deduced previously, so this value does not seem unreasonable if the ion damage strongly reduces the carrier density in the barrier.

It is clear from the results that our fabrication method which uses a FIB to remove a $50 \mathrm{~nm}$ wide trench from a track strongly modifies the remaining $\mathrm{MgB}_{2}$ by ion-damage and direct Ga-implantation. To our knowledge this is the first report of Josephson junctions in thin film $\mathrm{MgB}_{2}$. The high $I_{C} R_{N}$ product for the junctions fabricated in this way compared to other BCS-superconductor SNS junction techniques, coupled with the much higher $T_{C}$ make these junctions promising candidates for a range of applications.

\section{Acknowledgements}

This work was supported the UK Engineering and Physical Sciences, and Particle Physics and Astronomy Research Councils and the Korean Ministry of Science and Technology under the National Research Laboratory Project.

\section{Figure Captions}

Figure 1 Current-Voltage characteristics for 5, 13 and 21K. Inset, the resistance above $T_{C}$ of a track before placing in the FIB (dashed line), the same track after placing in the FIB (crosses), and the track in which the junction in the main figure was made, after the FIB stage (solid line).

Figure 2 Critical current-normal state resistance product against reduced temperature for the $\mathrm{MgB}_{2}$ device in Fig. 1 (left scale, solid circles) and a junction fabricated by focussed electron-beam irradiation in YBCO (right scale, open squares) ${ }^{25}$. Inset, normal state resistance of the $\mathrm{MgB}_{2}$ device reported here against reduced temperature.

Figure 3 Critical current for the same device as in previous figures against applied magnetic field and at $18 \mathrm{~K}$ : continuous line - increasing field, crosses - decreasing field.

Figure 4 Critical current (solid line, left scale), and height of the first (solid line) and second (dashed line) Shapiro steps against applied microwave power for the same device as in previous figures and at $11 \mathrm{~K}$. Inset, Current voltage characteristics for the device at three representative powers. 
1. Nagamatsu, J., Nakagawa, N., Muranaka, T., Zenitani, Y. \& Akimitsu, J. Superconductivity at $39 \mathrm{~K}$ in magnesium diboride. Nature 410, 63-64 (2001).

2. Canfield, P. C. et al. Superconductivity in dense MgB2 wires. Phys. Rev. Lett. 86, 2423-2426 (2001).

3. Larbalestier, D. C. et al. Strongly linked current flow in polycrystalline forms of the superconductor MgB2. Nature 410, 186-189 (2001).

4. Jin, S., Mavoori, H., Bower, C. \& van Dover, R. B. High critical currents in iron-clad superconducting MgB2 wires. Nature 411, 563-565 (2001).

5. Glowacki, B. A. et al. Superconductivity of powder-in-tube MgB2 wires. Supercond. Sci. Tech. 14, 193-199 (2001).

6. Schmidt, H., Zasadzinski, J. F., Gray, K. E. \& Hinks, D. G. Energy gap from tunneling and metallic contacts onto MgB2: Possible evidence for a weakened surface layer. Phys. Rev. B 63, 220504 (2001).

7. Sharoni, A., Felner, I. \& Millo, O. Tunneling spectroscopy and magnetization measurements of the superconducting properties of MgB2. Phys. Rev. B 63, 220508 (2001).

8. Szabó, P. et al. Evidence for two superconducting energy gaps in MgB2 by point-contact spectroscopy., Preprint cond-mat/0105598 at (xxx.lanl.gov) (2001).

9. Karapetrov, G., Iavarone, M., Kwok, W. K., Crabtree, G. W. \& Hinks, D. G. Scanning tunneling spectroscopy in MgB2. Phys. Rev. Lett. 86, 4374-4377 (2001).

10. Eom, C. B. et al. Thin Film Magnesium Boride Superconductor with Very High Critical Current Density and Enhanced Irreversibility Field. Nature 411, 558-560 (2001).

11. Kang, W. N., Kim, H. J., Choi, E. M., Jung, C. U. \& Lee, S. I. MgB2 superconducting thin films with a transition temperature of 39K. Science 292, 1521-1523 (2001).

12. Moon, S. H. et al. High critical current densities in superconducting MgB2 thin films., Preprint cond-mat/0104230 at (xxx.lanl.gov) (2001).

13. Wang, S. F. et al. Superconducting MgB2 Thin Films with Tc of about 39K Grown by Pulsed Laser Deposition., Preprint cond-mat/0104555 at (xxx.lanl.gov) (2001).

14. Blank, D. H. A. et al. Superconducting Mg-B films by pulsed-laser deposition in an in situ two-step process using multicomponent targets. Appl. Phys. Lett. in the press (2001). 
15. Brinkman, A. et al. Superconducting Quantum Interference Device based on MgB2 nanobridges., Preprint cond-mat/0105535 at (xxx.lanl.gov) (2001).

16. Latif, A., Booij, W. E., Durrell, J. H. \& Blamire, M. G. In-situ resistivity monitoring as an end-point detection technique in focused ion beam fabrication. J. Vac. Sci. Technol. B 18, 761-764 (2000).

17. Moseley, R. W., Tarte, E. J., Booij, W. E. \& Blamire, M. G. Direct writing of low Tc superconductor-normal metal-superconductor junctions using a focused ion beam. Appl. Phys. Lett. 75, 262-264 (1999).

18. Mitchell, E. E., Foley, C. P., Muller, K. H. \& Leslie, K. E. Vortex penetration and hysterestic behaviour of narrow planar Josephson junctions in a magnetic field. Physica C 321, 219-230 (1999).

19. Johansen, T. H. et al., Preprint cond-mat/0104113 at (xxx.lanl.gov) (2001) (2001).

20. Chen, X. H., Xue, Y. Y., Meng, R. L. \& Chu, C. W. Penetration Depth and Anisotropy in MgB2., Preprint cond-mat/0103029 at (xxx.lanl.gov) (2001).

21. Finnemore, D. K., Ostenson, J. E., Bud'ko, S. L., Lapertot, G. \& Canfield, P. C. Thermodynamic and transport properties of superconducting $\mathrm{Mg}^{10} \mathrm{~B}_{2}$. Phys. Rev. Lett. 86, 2420-2422 (2001).

22. Takano, Y. et al. Superconducting properties of MgB2 bulk materials prepared by high-pressure sintering. Appl. Phys. Lett. 78, 2914-2916 (2001).

23. Gonnelli, R. S. et al. Josephson effect in MgB_2 break junctions., Preprint cond-mat/0105241 at (xxx.lanl.gov) (2001).

24. Delin, K. A. \& Kleinsasser, A. W. Stationary properties of high-criticaltemperature proximity effect Josephson junctions. Supercond. Sci. Tech. $\mathbf{9}$, 227-269 (1996).

25. Booij, W. E., Pauza, A. J., Tarte, E. J., Moore, D. F. \& Blamire, M. G. Proximity coupling in high Tc Josephson junctions produced by focused electron beam irradiation. Phys. Rev. B 55, 14600 - 14609 (1997). 


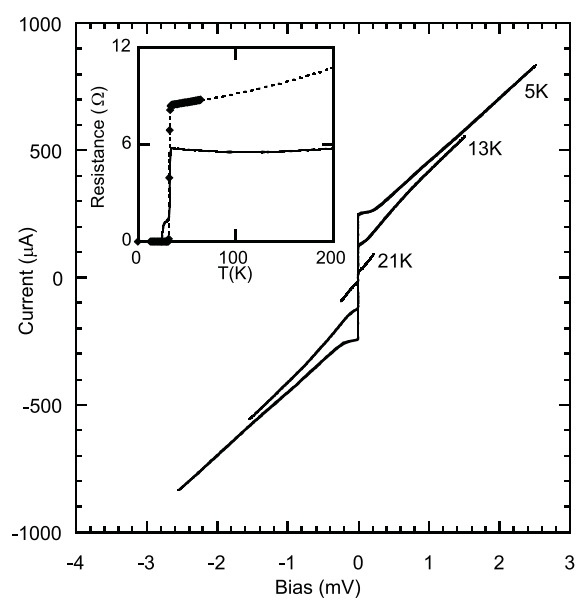

Burnell Figure 1

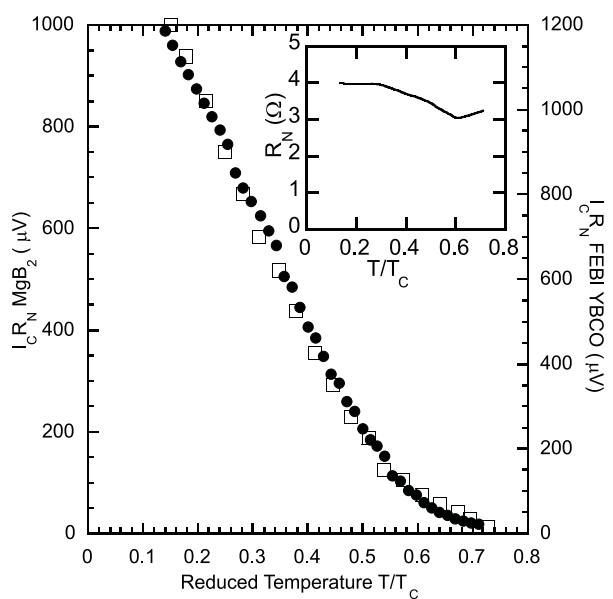

Burnell Figure 2 


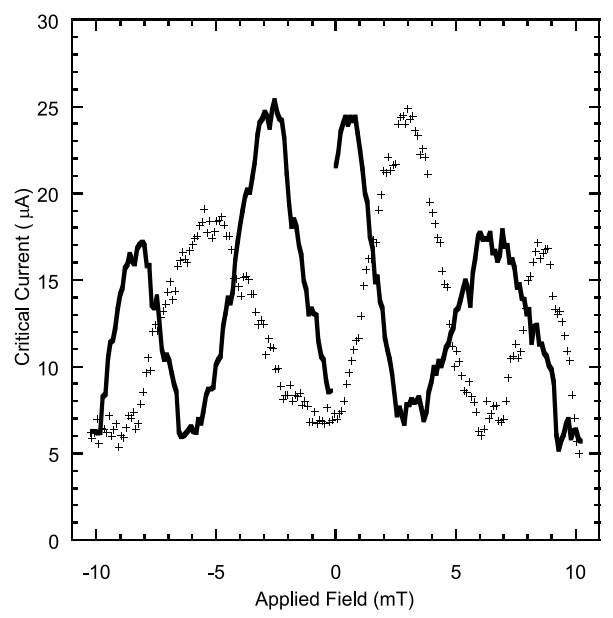

Burnell Figure 3

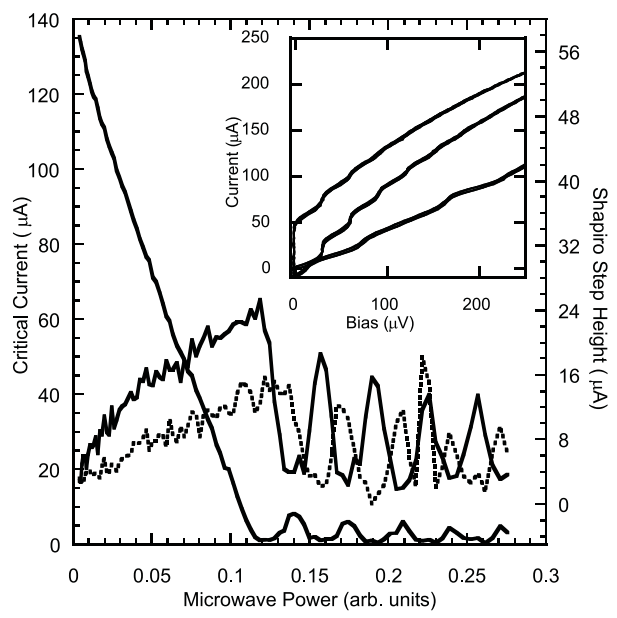

Burnell Figure 4 